\title{
Cualidades más valoradas en un residente por los tutores de una Unidad Docente de Medicina Familiar y Comunitaria
}

\author{
R. Sesma Arnáiz, J. Saura-Llamas*, J. Fernández Cuenca, A. Sáez Yanguas** \\ Especialista en Medicina Familiar y Comunitaria. *Tutor de Medicina Familiar y Comu - \\ nitaria **Residente de Mediana Familiar y Comunitaria. Centro de Salud \\ "Barrio del Carmen". Murcia
}

\section{RESUMEN}

Fundamento: desconocimiento de la opinión que tienen los tutores sobre los residentes.

Objetivo: conocer las cualidades que debe tener un residente de Medicina Familiar y Comunitaria $(M F y C)$ según la opinión de los tutores de la Uni dad Docente de Murcia.

Sujetos y método: estudio cualitativo, descripti vo. Sujetos: todos los tutores de los Centros de Salud docentes con residentes de MFyC, incluyendo médicos de familia y pediatras. Se realiza una en cuesta autoaplicable, repartida personalmente, anónima y de respuestas abiertas sobre la base de una única pregunta: ¿qué cualidades valoras más positivamente en un residente? Las respuestas se agruparán por similitud con los aspectos a valorar en un tutor según el programa docente de la espe cialidad (relación tutor-residente, cualificación clí nica, cualificación docente, actitud personal, tra bajo en equipo y de investigación y cualificación psicosocial). Los datos se presentan en cifras absolutas y porcentajes sobre el total de respuestas.

Resultados: se obtienen un total de 18 encues tas, con una media de más de nueve respuestas por tutor. Según el número total de respuestas del conjunto de los tutores la cualidad más valorada se refiere a la actitud personal del residente (un 41,2\%). A continuación figuran su cualificación docente (17,4\%), la cualificación clínica y los as pectos de la relación tutor-residente (13,9 y 13,4\% respectivamente). Son menos valoradas la cualifi cación psicosocial y de relación con los pacientes
Most valued qualities in residents according to the tu tors of a Family Community Medicine Teaching Unit

\begin{abstract}
Background: ignorance of tutor's opinion about their residents.

Objective: to know the qualities that Family and Community Medicine residents must have according to the opinion hold by tutors from the Teaching Unit in Murcia.

Subjects and method: descriptive, qualitative study. Subjects: all tutors from teaching ambulato ries with Family and Comunitary Medicine resi dents, including Family and Comunitary physi cians and pediatricians. An anonymous, self-applied, personally distributed, open-ans wered inquiry is made on the basis of only one question: which qualities do you value most positi vely in a resident? The answers were classified by items to be valued in a tutor according to the Spe ciality Program. Data are presented in absoluted numbers and in percentages over total numbers of answers.

Results: eighteen inquiries are obtained, with an average of more than nine answers per resi dent. Qualities most positively valued are related to the personal attitudes of the resident $141.2 \%$ of all answers). Next appear the teaching abilities (17.4\%), clinical qualifications and the ability of resident to communicate and get a good relation ship with the tutor (13.9 and $13.4 \%$, respectively). The less valued items are relationship with patients (8.1\%), capability of working as a part of a group and research abilities (less than 5\%). More than $50 \%$ of the answers are referred to the perso -
\end{abstract}

Aceptación: 19-07-01 
(8,1\%), y la capacidad de trabajo en equipo y de investigación (menos del 5\% cada una). Más del $50 \%$ del total de respuestas se refieren a la actitud personal del residente y a su cualificación docente.

Conclusiones principales: para estos tutores los grupos de cualidades más importantes que debe tener un residente de $M F y C$ se refieren a una acti tud personal positiva y su actitud ante la docencia. Estas opiniones pueden resultarnos muy valiosas para tratar de mejorar la formación de los resi dentes en el futuro.

Palabras clave: Medicina de Familia. Forma ción postgrado. Tutor. nal attitudes of the resident and with teaching ablilities.

Main conclusions: for these tutors, the most im portant qualities that the residents must have are their personal attitudes and teaching abilities. These opinions can be useful to try to improve the residents trainig in the future.

Key words: Family Medicine. Postgraduate stu dies. Tutor profiles.

\section{INTRODUCCIÓN}

La relación residente-tutor, o tutor-residente, desempeña un papel decisivo en la formación de los especialistas en medicina familiar y comunitaria (MFyC), subrayando su importancia tanto el programa docente de la especialidad ${ }^{1-3}$ como la opinión de distintos autores ${ }^{4-7}$. Así el programa docente establece una relación individualizada entre tutor y residente, al objeto de conseguir una formación de calidad.

Esta relación es el elemento clave de los resultados que vamos a obtener y afortunadamente cada día son más frecuentes las aportaciones que se van haciendo a este tema, aunque desde distintos puntos de vista. Así conocemos cómo debe ser un tutor (T) desde el punto de vista institucional y según la opinión de distintos grupos de expertos ${ }^{8}$.

Conocemos la normativa legal que deben cumplir los residentes, así como los conocimientos, habilidades y actitudes que deben adquirir, y la evaluación que deben realizar para superar su periodo formativo y obtener el título de especialista en Medicina Familiar y Comunitaria; pero la Comisión Nacional de la Especialidad (CNE) no hace referencia a las cualidades o requisitos que deben reunir los residentes.

También sabemos que existe un cierto grado de insatisfacción de los MIR $^{9,10}$, aunque el colectivo de residentes de MF valora de una manera global y generalmente positiva, distintos aspectos de su periodo de especialización ${ }^{11}$.

A pesar de todo lo anterior la información existente sobre los residentes es muy limitada, pues aunque en muchas Unidades Docentes existen encuestas y evaluaciones recogidas durante años habitualmente, éstas no se han publicado; y además se han realizado a posteriori, es decir, una vez que el proceso formativo ya ha tenido lugar (sin tener por tanto capacidad de intervención).
Asumiendo la necesidad de la participación de los tutores a lo largo de todo el proceso formativo ${ }^{12,13}$, es necesario recabar directamente su opinión, recogiéndola a priori (antes de que el proceso tenga lugar), sobre las características que debe tener el residente, ya que el mayor conocimiento del proceso formativo y de las expectativas de los tutores sobre los residentes pueden aportar efectos beneficiosos para ellos y para las Unidades Docentes. Nuestro objetivo es conocer las cualidades que debe tener un Residente de MFyC según la opinión de los tutores de la Unidad Docente de Murcia; comparándolo con el perfil que para el tutor establece el programa docente de la especialidad.

Siguiendo una línea de trabajo sobre la docencia postgrado en Medicina Familiar y Comunitaria ${ }^{14-18}$, y completando un estudio previo realizado con la opinión los residentes sobre las cualidades que debe tener un tutor, se exponen a continuación los resultados de una encuesta de opinión realizada a los tutores en el año 1999.

\section{MATERIAL Y MÉTODOS}

Se trata de un estudio cualitativo, descriptivo, mediante la realización de una encuesta autoaplicable, repartida personalmente, anónima y de respuestas abiertas, sobre la base de una única pregunta: “¿qué cualidades valoras más positivamente en un residente?".

Se ha realizado con los tutores de los Centros de Salud docentes con residentes de MFyC, incluyendo médicos de familia y pediatras. Las encuestas se entregaron en abril de 1999. En total se repartieron 32 cuestionarios, de los que se han obtenido ${ }^{18}$ encuestas cumplimentadas.

Para la administración de la encuesta se elaboró un dossier conteniendo el cuestionario y las instruc- 
ciones para su correcta cumplimentación, así como un sobre en el que depositar la encuesta contestada y enviar por correo a nuestro Centro de Salud. Se entregó este dossier a cada uno de los residentes para que éstos se lo hicieran llegar a sus tutores correspondientes; o bien se le dio directamente uno de los miembros del grupo de investigación. Se recogieron los cuestionarios cumplimentados durante dos meses. Se realizó una recaptación al mes de finalizar la primera recogida. Los casos perdidos son los cuestionarios no devueltos.

Se agruparon las respuestas por similitud con un artículo anterior ${ }^{19}$ y el proceso de acreditación establecido por el programa docente ${ }^{2}$. Esta forma de agrupar los resultados nos permite establecer comparaciones entre ambos trabajos. Así, los aspectos que hay que valorar en un tutor y en un residente son: cualificación clínica, cualificación docente, cualificación psicosocial y de relación con los pacientes, capacidad de investigación, de trabajo en equipo, y de mantener una adecuada relación tutorresidente. Se añadieron, además, los apartados de actitud personal del residente y otras cualidades, que aparecieron como nuevos grupos de respuestas en el trabajo anterior.

Los datos se presentan en cifras absolutas y porcentajes sobre el total de encuestas y de respuestas, priorizando los distintos grupos de cualidades según el porcentaje obtenido sobre el total de respuestas.

\section{RESULTADOS}

Del total de 32 encuestas repartidas contestaron 18 tutores (un 56,25\%), con un total de 172 respuestas diferentes, y con una media de más de nueve respuestas por tutor. En la tabla I se detallan los resultados globales así como la priorización de los

\section{Tabla I}

PRIORIZACIÓN DE LOS GRANDES GRUPOS DE CUALIDADES

QUE DEBE TENER UN RESIDENTE SEGÚN EL TOTAL DE RESPUESTAS DEL CONJUNTO DE LOS TUTORES

\begin{tabular}{lcc}
\hline Cualidades & $\begin{array}{c}N^{\circ} \text { total de } \\
\text { respuestas }\end{array}$ & $\begin{array}{c}\text { \% sobre el total } \\
\text { de respuestas }\end{array}$ \\
\hline Actitud personal & 71 & 41,2 \\
Cualificación docente & 30 & 17,4 \\
Cualificación clínica & 24 & 13,95 \\
Relación tutor-residente & 23 & 13,37 \\
Cualificación psicosocial & 14 & 8,13 \\
Trabajo en equipo & 8 & 4,65 \\
Investigación & 2 & 1,16 \\
\hline Total & 172 & 100 \\
\hline
\end{tabular}

grandes grupos de cualidades que debe tener un residente según la opinión de los tutores.

Considerando cada cualidad de forma aislada y el número de tutores que la han mencionado (relacionando veces que se repite una respuesta con el total de encuestas contestadas), 12 de ellos (un $66 \%$ de los tutores) señalan el interés del residente por aprender, mientras que las cualidades: que le guste la Medicina de Familia, que tengan iniciativa propia, una buena formación previa, capacidad de estudio, y buen trato con el paciente son señaladas por 5 tutores (el 28\% de ellos). El resto de cualidades son mencionadas por un número menor de tutores.

Considerando cada cualidad de forma aislada y el número de veces que se ha mencionado (priorizándolas según el número de veces que se repite en relación al total de respuestas), los datos son similares a los anteriores (Tabla II).

Según el número total de respuestas del conjunto de los tutores el grupo de cualidades más valoradas se refiere a la actitud personal del residente (con un $41,2 \%$ del total de respuestas). A continuación figuran su cualificación docente $(17,4 \%)$, la cualificación clínica y los aspectos de la relación tutorresidente (13,9 y $13,4 \%$, respectivamente). Son menos valoradas la cualificación psicosocial y de relación con los pacientes $(8,1 \%)$, y la capacidad de trabajo en equipo y de investigación (menos del $5 \%$ cada una) (Tabla I).

En conjunto, más del $50 \%$ del total de las respuestas se refieren a la suma de la actitud personal del residente y su cualificación docente.

Para ilustrar con más exactitud el contenido cualitativo de las opiniones de los tutores, vamos a detallar algunas de ellas en los apartados más valorados:

-En cuanto a la actitud personal del residente algunas de las respuestas más repetidas fueron: "que le guste lo que hace, la medicina de familia", "que sea responsable", "que sea puntual", "que sea humilde", "que tenga buen humor", "que sea prudente en la toma de decisiones", "que tenga cualidades humanas", "que tenga disponibilidad temporal", "que sea participativo", "que sea tolerante", "que sea respetuoso", "que tenga ganas de trabajar", "que sea entusiasta", "que sea decidido".

- Respuestas relacionadas con la cualificación docente: "que tenga interés por aprender", "que tenga iniciativa propia", "que sea capaz de asumir tareas y responsabilidades paulatinamente", "que tenga motivación e ilusión por el aprendizaje", "que le guste la lectura científica".

—Respecto a la cualificación clínica: "que tenga los conocimientos clínicos suficientes", "que sea capaz de estudiar para resolver los casos que se planteen en la consulta", "que tenga capacidad de 
Tabla II

PRIORIZACIÓN DE LAS CUALIDADES MÁS VALORADAS EN UN RESIDENTE SEGÚN LA IMPORTANCIA DE UNA RESPUESTA EN RELACIÓN AL TOTAL DE RESPUESTAS (SE INCLUYEN SÓLO LAS CUALIDADES QUE HAN OBTENIDO MÁS DE DOS RESPUESTAS)

\begin{tabular}{|c|c|c|c|}
\hline $\begin{array}{l}\text { Grupos de } \\
\text { cualidades }\end{array}$ & Cualidades & $\begin{array}{l}\mathrm{N}^{\circ} \text { de } \\
\text { respuestas }\end{array}$ & $\begin{array}{l}\% \text { del total de } \\
\text { respuestas }\end{array}$ \\
\hline Actitud personal & $\begin{array}{l}\text { Que le guste la Medicina de Familia } \\
\text { Responsable } \\
\text { Puntual } \\
\text { Humilde }\end{array}$ & $\begin{array}{l}5 \\
4 \\
3 \\
3\end{array}$ & $\begin{array}{l}2,90 \\
2,32 \\
1,74 \\
1,74\end{array}$ \\
\hline Cualificación docente & $\begin{array}{l}\text { Interés por aprender } \\
\text { Iniciativa propia } \\
\text { Asumir responsabilidades de forma } \\
\text { paulatina } \\
\text { Motivación }\end{array}$ & $\begin{array}{c}12 \\
5 \\
5 \\
2\end{array}$ & $\begin{array}{l}6,97 \\
2,90 \\
2,90 \\
1,16\end{array}$ \\
\hline Cualificación clínica & $\begin{array}{l}\text { Formación previa } \\
\text { Capacidad de estudio } \\
\text { Capacidad de trabajo } \\
\text { Implicación en mejorar sus conocimientos }\end{array}$ & $\begin{array}{l}5 \\
5 \\
4 \\
3\end{array}$ & $\begin{array}{l}2,90 \\
2,90 \\
2,32 \\
1,74\end{array}$ \\
\hline Relación tutor-residente & $\begin{array}{l}\text { Empatía } \\
\text { Cordialidad con el tutor } \\
\text { Capaz de preguntar sus dudas } \\
\text { Naturalidad con el tutor }\end{array}$ & $\begin{array}{l}3 \\
2 \\
2 \\
2\end{array}$ & $\begin{array}{l}1,74 \\
1,16 \\
1,16 \\
1,16\end{array}$ \\
\hline Cualificación psicosocial & $\begin{array}{l}\text { Buen trato al paciente } \\
\text { Humanidad con los pacientes }\end{array}$ & $\begin{array}{l}5 \\
3\end{array}$ & $\begin{array}{l}2,90 \\
1,74\end{array}$ \\
\hline Trabajo en equipo & $\begin{array}{l}\text { Capacidad de trabajar en equipo } \\
\text { Integración con el equipo }\end{array}$ & $\begin{array}{l}2 \\
2\end{array}$ & $\begin{array}{l}1,16 \\
1,16\end{array}$ \\
\hline Investigación & Que tenga interés investigador & 2 & 1,16 \\
\hline
\end{tabular}

trabajo clínico", "que se implique en mejorar sus conocimientos", "que tenga capacidad de decidir", "que sea profesional".

- Acerca de la relación tutor-residente: "que haya empatía", "que el residente sea capaz de preguntar sus dudas", "que sea una relación cordial", "que el residente sea natural con el tutor, que se muestre tal y cómo es", "que tenga confianza con el tutor". "que haya comunicación entre los dos".

Para la presentación de los resultados cualitativos de este estudio (recogiendo textualmente su expresión) y dada su amplitud, se han seleccionado aquellas respuestas con más de una contestación para cada cualidad (Tabla II).

\section{DISCUSIÓN}

Hasta este momento el colectivo de tutores ha reflexionado poco, o ha publicado poco, sobre el residente de Medicina de Familia ${ }^{20}$. Este estudio nos permite conocer, al menos parcialmente, cuáles son las expectativas de los tutores respecto a las cualidades que debe tener un residente.

La pregunta en la que se basa la encuesta es clara y directa, por lo que creemos no presenta problemas de interpretación; aunque los resultados obtenidos de una sola pregunta tan general impiden extraer conclusiones generalizables, no impiden su utilidad. Se decidió realizar una pregunta tan abierta a fin de facilitar la máxima libertad de expresión a los tutores.

El número total de encuestas recibidas 18 , no es grande, pero está en relación al número total de tutores de la Unidad Docente, ámbito elegido para la realización del estudio.

Del total de encuestados (18), 2 fueron recaptados por contacto personal directo 1 mes después, tras fallar su respuesta en un primer intento. Aunque el porcentaje de participación fue de más del $56 \%$, que consideramos que es una tasa aceptable para encuestas generales de este tipo, esperábamos una tasa de respuesta más alta. Esta tasa puede ser consecuencia del 
cansancio de los tutores ante la multitud de colaboraciones similares que se les han solicitado ( sin darles luego la retroinformación adecuada). Probablemente al solicitarse opinión en un sentido positivo (descripción de un buen residente) la influencia de la tasa de respuesta no es muy significativa.

Se obtuvo un número elevado de respuestas por encuesta (una media de 9,5 por participante), lo que nos indica que los tutores han reflexionado sobre los aspectos a valorar en el residente.

Asumimos como posible sesgo la inclusión subjetiva de las respuestas en uno u otro epígrafe, aunque han sido debatidas ampliamente por el grupo de investigación.

La cualidad más valorada por los tutores se refiere al "interés del residente por aprender", siendo destacada por el $66,6 \%$ de ellos.

Los tutores han insistido mucho en las cualidades humanas y personales de los residentes, dando mucha importancia a que al residente le guste la medicina de familia y a que se comporte con responsabilidad y prudencia, no sólo desde el punto de vista clínico, sino también desde el punto de vista personal.

Nos llama la atención que la cualificación clínica no sea uno de los grupos de valores más deseables en los residentes (sólo con un 13,9 \% de respuestas), no refiriéndose ninguna de ellas al expediente académico; sin embargo sí son valoradas tanto la capacidad de trabajo como de estudio, y la resolución de problemas clínicos. Esto puede indicarnos cierta preocupación de los tutores ante la necesidad compartir sus pacientes con otra persona (el residente), y de delegar en ellos la responsabilidad de su consulta. Por otro lado se tiene en cuenta que los residentes aún están aprendiendo, y así los tutores parecen aceptar sus limitaciones como clínicos y valorar sus avances en el ámbito formativo durante los tres años de la residencia.

Parece que preocupan proporcionalmente menos los aspectos de comunicación y de relación personal con el residente; ya que aunque valoran que éste sea cordial, que se comporte con naturalidad y con respeto hacia su tutor; y aunque parece implícito, en ninguna respuesta se hace mención explícita al establecimiento de una relación personal de mutua confianza y buena comunicación.

Comparando los resultados obtenidos de este estudio y los obtenidos en el trabajo anterior ${ }^{19}$, destaca que mientras los tutores valoran en primer lugar la actitud personal de los residentes, a éstos les preocupaba más la relación interpersonal (un 35,5\% de respuestas). Para los residentes la cualificación docente de los tutores queda relegada a una cuarta posición, a pesar de que debería preocuparles más, ya que ésta es esencial en un tutor.
En lo referente a la cualificación clínica, los residentes quieren que su tutor sea un buen clínico, resolutivo y que tenga experiencia, lo que puede traducir la necesidad de un modelo Médico de Familia que comparta con ellos su sabiduría y experiencia. Esto resulta coherente con los roles que desempeñan cada uno de ellos ${ }^{12}$.

Es destacable el hecho de que en ambos estudios, es decir, tanto para los residentes como para los tutores, los aspectos menos valorados fueron la cualificación psicosocial (en la que ambos señalan la importancia del buen trato a los pacientes y la humanidad con ellos), y el trabajo en equipo y de investigación; lo cual podría explicarse por el hecho de que estos aspectos suponen la participación de un tercer elemento u otros actores (pacientes, equipo) en la relación entre tutor y residente.

Al tratar de comparar nuestro estudio con otros similares, nos encontramos con la ausencia de referencias en castellano y en los países de nuestro entorno ${ }^{21-23}$. Existen varias publicaciones acerca de la formación postgrado ${ }^{14,24}$ y sobre la relación entre tutores y residentes y el proceso de tutorización ${ }^{25-27}$, pero no hemos encontrado estudios en los que se analicen las dimensiones que más valoran los tutores de los residentes.

Con la información recogida de este estudio, podemos concluir que en el "perfil del residente ideal" de Medicina Familiar y Comunitaria para los tutores de la Unidad Docente de Murcia lo fundamental es que el residente tenga una actitud personal positiva hacia la MF, hacia la docencia, y que tenga mucho interés por aprender. A la vista de estos resultados surgen algunas preguntas: ¿todas las cualidades aquí descritas, para el residente, son exigibles?, ¿la mayoría son esperables? A pesar de que estemos en el terreno de lo opinable, las cualidades (o características) que estos tutores quieren en los residentes parecen muy razonables ya que son probablemente esperables y deseables; siendo muy difícil tratar de ayudar a formar a personas sin interés por aprender y con una actitud negativa en el terreno personal y en el de la formación.

Parece además que estos valores: una actitud general positiva y hacia el ejercicio profesional y un elevado interés en seguir aprendiendo, serían definitorios del perfil profesional del Médico de Familia ${ }^{1,28}$; y probablemente exigibles desde el punto de vista ético ${ }^{29}$.

Esta reflexión puede ayudarnos a conocer mejor las expectativas de los tutores, acercándonos al que debería ser el perfil adecuado del residente, y así poder avanzar en el diseño de un nuevo programa docente que estimulara la adquisición de estas habilidades y actitudes, así como establecer nuevos criterios de acceso a la formación especializada, a 
la evaluación de los residentes ${ }^{30}$, o incluso a la evaluación de los Médicos de Familia.

Pero, ¿qué hacemos con los residentes cuyas cualidades sean distintas de las deseadas por los tutores? Probablemente para tratar de resolver este problema los tutores deberíamos adquirir más y mejor metodología docente (conocimientos, habilidades y actitudes docentes).

En un futuro inmediato sería preciso realizar estudios similares o publicar los datos ya existentes en otras Comunidades Autónomas y Unidades Docentes, a fin de mejorar nuestros conocimientos sobre el tema y poder compararlos. Además, sería conveniente seguir investigando sobre la formación de los residentes de MFyC para conocer todos aquellos aspectos que influyen en su formación, incluyendo el proceso formativo: métodos de tutorización, tiempo de dedicación al residente, rotaciones hospitalarias y en el Centro de Salud, guardias hospitalarias,...para identificar las deficiencias docentes e intentar solventarlas.

\section{AGRADECIMIENTOS}

Agradecemos la colaboración a todos los tutores y residentes que han participado en este estudio, así como a los residentes y tutores del Centro de Salud del Barrio del Carmen.

\section{CORRESPONDENCIA:}

José Saura Llamas

$\mathrm{C} /$ Atenea $\mathrm{n}^{\circ} 21$.

30120 El Palmar. Murcia

Fax: 968-268091.

e-mail: csgoya@iname.com

\section{Bibliografía}

1. Comisión Nacional de la Especialidad de Medicina Familiar y Comunitaria. Medicina Familiar y Comunitaria. En: Guía de Formación de Especialistas. $3^{\mathrm{a}}$ ed. Madrid: Ministerio de Sanidad y Consumo, 1996.

2. Programa docente de la Especialidad de Medicina Familiar y Comunitaria. Madrid: Ministerio de Sanidad y Consumo. Ministerio de Educación y Cultura, 1993.

3. Ministerio de Sanidad y Consumo. Guía de formación de Especialistas. Madrid: Secretaria General Técnica, 1996.

4. Forés García D. ¿Son válidos los criterios actuales de acreditación docente de los centros y tutores de la especialidad de medicina familiar y comunitaria? FMC 1996; 3: 383-90.

5. Gayoso Diz P, Viana Zulaica C, García González J, Tojal del Casero F. Los tutores de medicina de familia: actitudes y actividades de tutorización. Aten Primaria 1995; 15: 481-6.

6. Gallo Vallejo FJ, editor. Manual del Residente de Medicina Familiar y Comunitaria. Madrid: Lab. Beecham, 1993.

7. Martín Zurro A, Cano Pérez JF. Atención Primaria. Conceptos, Organización y Práctica Clínica. $3^{\mathrm{a}}$ ed. Barcelona: Mosby, 1994.

8. Saura Llamas J. El Tutor de Atención Primaria. Aten Primaria 1993; 11: 273-4.

9. Pujol Farriols R. Médicos residentes (MIR) insatisfechos. Med Clin (Barc) 1997; 109: 623-4.

10. Fernández-Llamazares J, Julián JF, Hidalgo F, García $F$, Moreno P, Vega JL, et al. Encuesta a residentes MIR sobre su satisfacción tras obtener una plaza hospitalaria. Med Clin (Barc) 1997; 109: 615-8

11. Thomas Mulet V, Puig Valls B, Lloveras Canaves J, Pareja Bezares A. El residente de medicina familiar y comunitaria ante su especialización (II). Rev San Hig Púb 1991; 65: 7785.

12. Pelayo Álvarez M, Gutiérrez Sigler L, Albert Ros X. Influencia del perfil profesional de los tutores de medicina familiar y comunitaria en la formación de sus residentes. Aten Primaria 1998; 21:145-54.
13. Leal Hernández M, Saura Llamas J, López Piñera M, De la Viesca Cosgrove S, Ferrer Mora A, García Méndez MM. Investigación en la acción. Cómo facilitar la incorporación de los residentes de MF y $\mathrm{C}$ al centro de salud. Aten Primaria 1996; 18: 571-6.

14. Saura Llamas J, Quirós Bauset C, Molina Durán F. Docencia postgrado: ¿Cómo llevar a cabo la tutorización de residentes de medicina familiar y comunitaria? Aten Primaria 1994; 13 : 103-4.

15. León Martínez LP, Gómez Jara P, Martínez González-Moro N, Navarro Martínez A, Guillén Montiel C, Saura Llamas J. La formación posgraduada en Medicina Familiar y Comunitaria. La importancia de las diferencias entre la actividad asistencial del tutor y del residente. Aten Primaria 1994; 14 : 661-4.

16. Saura Llamas J, Leal Hernández M, García Méndez MM, López Piñera M, Ferrer Mora A, de la Viesca Cosgrove S. Motivos de ansiedad en los profesionales de atención primaria. Comparación residentes - tutores. Aten Primaria 1997; 20: $142-6$.

17. Saura Llamas J, Leal Hernández M. Docencia postgrado: factores condicionantes de la relación tutor - residente en medicina familiar. Aten Primaria 1997; 20: 511-8.

18. Saura Llamas J. Momentos clave en la formación del Médico de Familia. Dimens Hum 1998; 2 (2): 53-7.

19. Saura Llamas J, Martínez Carmona I, Leal Martínez M, Sesma Arnáiz R, Bernal Lajusticia M. Cualidades más valoradas en un tutor por los residentes de una Unidad Docente de Medicina Familiar y Comunitaria. Medifam 1999; 9: 359-66.

20. Fernández Sierra J. El curriculum de los MIR de Medicina Familiar y Comunitaria. Reflexiones desde la pedagogía. Medifam 1995; 5 (6): 345-50.

21. Aleomoni LM. Student ratings of instruction. En: Millman J, ed. Handbook of Teacher Evaluation. Beverly Hills, California: Sage Publications, 1981. 
22. Irby DM, Gillmore GM, Ramsey PG. Factors affecting ratings of clinical teachers by medical students and residents. J Med Education 1987; 1-7.

23. McLeod PJ, James CA, Abrahamowicz M. Clinical tutor evaluation; a 5-year study by students on a in patient service and residents in an ambulatory care clinic. Medical Education 1993; 27: 28-54.

24. Martin Zurro A. La formación postgraduada en medicina de familia en España- Jano 1996; L (1169): 1941.

25. Humphreys H. Recommendations to regions for the establishmen of criteria for the approval and reapproval of trainers in general practice. Londres: The Joint Commitee on Postgraduate Training for General Practitioners, 1993.

26. Euract. Vocational training in family medicine. How can we prepare the best family physicians? Budapest: Symposium 225th Aniversary of Semmelweis University, 1994.

27. Schwenk Thomas L, Whitman N. The Physician as Teacher. Baltimore: Williams Wilkins, 1987.

28. Gallo Vallejo FJ, Altisent Trota R, Díez Espino J, Fernández Sánchez C, Foz i Gil G, Granados Menéndez MI, et al. Perfil profesional básico del médico de familia. Barcelona: semFYC, 1994.

29. Altisent Trota R, Delgado Marroquín MT, Jolin Garijo L, Martín Espíldora MN, Ruiz Moral R, Simón Laborda P, et al. Sobre bioética y medicina de familia. Barcelona: semFYC, 1996.

30. Santos Guerra MA, Prados Torres JD, Fernández Sierra J, Martín Ruiz MV, Angulo Rasco F. Evaluación Externa de la Formación de Médicos Residentes: El arte de mejorar a través del conocimiento. Barcelona: semFYC, 1996. 